\title{
ACCURATE RATE CONSTANTS FOR THE FORWARD AND REVERSE H + CO $\leftrightarrow$ HCO REACTIONS AT THE HIGH-PRESSURE LIMIT
}

\author{
Rafael M. Vichietti, ${ }^{* 1}$ Francisco B. C. Machado, ${ }^{* 1}$ and Roberto L. A. Haiduke ${ }^{* 2}$
}

1. Instituto Tecnológico de Aeronáutica, Departamento de Química, Praça Marechal Eduardo Gomes, 50, Vila das Acácias, São José dos Campos, S.P., Brazil, CEP 12228-900, phone 55-123947-5957

2. Universidade de São Paulo, Instituto de Química de São Carlos, Departamento de Química e Física Molecular, Avenida Trabalhador São Carlense, 400, Parque Arnold Schimidt, São Carlos, S.P., Brazil, CEP 13566-590, phone 55-16-3373-9975

\section{SUPPORTING INFORMATION}

Table S1. Equilibrium bond lengths (in Angstrom), $r$, bond angles (in degree), $\theta$, and $T_{1}$ diagnostic for the molecular structures of stationary points along the $\mathrm{H}+\mathrm{CO} \leftrightarrow \mathrm{HCO}$ reaction. $^{\mathrm{a}}$

\begin{tabular}{|c|c|c|c|c|c|}
\hline Molecule & Parameters & $\begin{array}{c}\text { CCSD/ } \\
\text { aug-cc-pVTZ } \\
\text { (this work) }\end{array}$ & $\begin{array}{l}\operatorname{UCCSD}(\mathrm{T}) / \\
\operatorname{CBS}^{(1)}\end{array}$ & $\begin{array}{c}\text { MRCI+Q/ } \\
\text { cc-pCV5Z-DK }\end{array}$ & Experimental $^{\mathrm{b}}$ \\
\hline \multirow[t]{2}{*}{$\mathrm{CO}$} & $r(\mathrm{C}-\mathrm{O})$ & $1.1288[<0.1]$ & $\ldots$ & $\ldots$ & $1.1283^{(3)}$ \\
\hline & $T_{1}$ & 0.018 & $\ldots$ & $\ldots$ & $\ldots$ \\
\hline \multirow[t]{4}{*}{$\operatorname{PrC}$} & $r(\mathrm{H}-\mathrm{C})$ & 3.5888 & $\ldots$ & $\ldots$ & $\ldots$ \\
\hline & $r(\mathrm{C}-\mathrm{O})$ & 1.1288 & $\ldots$ & $\ldots$ & $\ldots$ \\
\hline & $\theta(\mathrm{H}-\mathrm{C}-\mathrm{O})$ & 98.7 & $\ldots$ & $\ldots$ & $\ldots$ \\
\hline & $T_{1}$ & 0.017 & $\ldots$ & $\ldots$ & $\ldots$ \\
\hline \multirow[t]{4}{*}{ TSRP } & $r(\mathrm{H}-\mathrm{C})$ & 1.8461 & 1.865 & 1.8467 & $\ldots$ \\
\hline & $r(\mathrm{C}-\mathrm{O})$ & 1.1336 & 1.133 & 1.1330 & $\ldots$ \\
\hline & $\theta(\mathrm{H}-\mathrm{C}-\mathrm{O})$ & 117.1 & 116.0 & 117.2 & $\ldots$ \\
\hline & $T_{1}$ & 0.022 & $\ldots$ & $\ldots$ & $\ldots$ \\
\hline \multirow[t]{4}{*}{$\mathrm{HCO}$} & $r(\mathrm{H}-\mathrm{C})$ & $1.1171[0.2]$ & $1.119[<0.1]$ & $1.1161[0.3]$ & $1.1191^{(4)}$ \\
\hline & $r(\mathrm{C}-\mathrm{O})$ & $1.1755[<0.1]$ & $1.175[<0.1]$ & $1.1756[<0.1]$ & $1.1754^{(4)}$ \\
\hline & $\theta(\mathrm{H}-\mathrm{C}-\mathrm{O})$ & $125.0[0.4]$ & $124.6[0.1]$ & $124.6[0.1]$ & $124.43^{(4)}$ \\
\hline & $T_{1}$ & 0.022 & $\ldots$ &.. & $\ldots$ \\
\hline
\end{tabular}

${ }^{\mathrm{a}}$ The values between brackets are absolute deviations (in \%) in relation to experimental data.

${ }^{\mathrm{b}}$ Experimental data for equilibrium geometries (from $r_{\mathrm{e}}$ structures). 
Table S2. Scaled fundamental vibrational frequencies $\left(\right.$ in $\mathrm{cm}^{-1}$ ) for the molecular structures of stationary points along the $\mathrm{H}+\mathrm{CO} \leftrightarrow \mathrm{HCO}$ reaction along with other anharmonic theoretical results and experimental data. ${ }^{\mathrm{a}}$

\begin{tabular}{ccccc}
\hline Molecule & $\begin{array}{c}\text { CCSD/ } \\
\text { aug-cc-pVTZ } \\
\text { this work) }\end{array}$ & $\begin{array}{c}\text { UCCSD(T)/ } \\
\text { CBS }^{(1)}\end{array}$ & $\begin{array}{c}\text { MRCI+Q/ } \\
\text { cc-pCV5Z-DK }\end{array}$ & Experimental \\
\hline CO & $2117.6[1.2]$ & $\ldots$ & $\ldots$ & $2143^{(5)}$ \\
PrC & 11.0 & $\ldots$ & $\ldots$ & $\ldots$ \\
& 63.0 & $\ldots$ & $\ldots$ & $\ldots$ \\
& 2117.5 & $\ldots$ & $\ldots$ & $\ldots$ \\
TSRP & $799.1 i^{\text {b }}$ & $\ldots$ & $\ldots$ & $\ldots$ \\
& 377.1 & $\ldots$ & $\ldots$ & $\ldots$
\end{tabular}

$\begin{array}{lllll}\text { HCO } & 1075.2[0.5] & 1079.6[0.1] & 1085.9[0.5] & 1081^{(3)} \\ & 1854.9[0.7] & 1871.6[0.2] & 1869.1[0.1] & 1868^{(3)} \\ & 2620.3[5.4] & 2428.5[2.3] & 2436.8[1.9] & 2485^{(3)}\end{array}$

${ }^{\text {a }}$ The values between brackets are absolute deviations (in \%) in relation to experimental data.

${ }^{\mathrm{b}}$ The symbol $i$ represents the imaginary frequency.

Table S3. Enthalpies (in kcal $\left.\mathrm{mol}^{-1}\right)$ relative to reactants $(\mathrm{H}+\mathrm{CO}$ ) for the stationary point structures associated with the $\mathrm{H}+\mathrm{CO} \leftrightarrow \mathrm{HCO}$ reaction, as calculated at the CCSD(T)/CBS//CCSD/aug-cc-pVTZ level and different temperatures (in K), $T$.

\begin{tabular}{rrrccc}
\hline \multicolumn{1}{c}{$T$} & PrC & TSRP & HCO & $\Delta H_{\exp }{ }^{\mathrm{a}}$ & $\Delta H_{\text {theor }}{ }^{\mathrm{b}}$ \\
\hline 50 & -0.07 & 3.48 & -14.60 & $\ldots$ & -14.9 \\
100 & -0.08 & 3.28 & -14.80 & -14.52 & -15.0 \\
200 & -0.09 & 2.96 & -15.20 & -14.91 & -15.5 \\
300 & -0.09 & 2.69 & -15.58 & -15.29 & -15.8 \\
500 & -0.09 & 2.24 & -16.23 & -15.94 & -16.4 \\
700 & -0.09 & 1.82 & -16.73 & -16.44 & -16.9 \\
1000 & -0.09 & 1.22 & -17.28 & -16.96 & -17.5 \\
1500 & -0.10 & 0.22 & -17.84 & -17.49 & -18.1 \\
2000 & -0.10 & -0.77 & -18.17 & -17.81 & -18.3 \\
2500 & -0.10 & -1.76 & -18.38 & -18.02 & -18.5 \\
3000 & -0.10 & -2.76 & -18.53 & -18.16 & -18.7 \\
3500 & -0.10 & -3.75 & -18.64 & -18.25 & -18.8 \\
4000 & -0.10 & -4.74 & -18.72 & -18.30 & -18.8 \\
\hline
\end{tabular}

${ }^{a}$ Experimental enthalpy changes for the $\mathrm{H}+\mathrm{CO} \leftrightarrow \mathrm{HCO}$ reaction retrieved from Chase (1998). ${ }^{(6)}$

${ }^{b}$ Theoretical enthalpy changes calculated from the ROCCSD(T)/CBS//UCCSD/cc-pVDZ combined treatment by Vichietti et al. (2019). ${ }^{(7)}$ 
Table S4. Gibbs energies (in $\left.\mathrm{kcal} \mathrm{mol}^{-1}\right)$ relative to reactants $(\mathrm{H}+\mathrm{CO})$ for the stationary point structures related to the $\mathrm{H}+\mathrm{CO} \leftrightarrow \mathrm{HCO}$ reaction, as calculated at the $\mathrm{CCSD}(\mathrm{T}) / \mathrm{CBS} / / \mathrm{CCSD} /$ augcc-pVTZ level and different temperatures (in K), $T$.

\begin{tabular}{rrrrrr}
\hline \multicolumn{1}{c}{$T$} & PrC & TSRP & \multicolumn{1}{c}{ HCO } & $\Delta G_{\exp }{ }^{\mathrm{a}}$ & $\Delta G_{\text {theor }}{ }^{\mathrm{b}}$ \\
\hline 50 & 0.20 & 4.12 & -13.90 & $\ldots$ & -14.1 \\
100 & 0.48 & 4.83 & -13.13 & -12.85 & -13.4 \\
200 & 1.04 & 6.51 & -11.30 & -11.02 & -11.6 \\
300 & 1.61 & 8.35 & -9.26 & -9.00 & -9.5 \\
500 & 2.75 & 12.24 & -4.87 & -4.61 & -5.1 \\
700 & 3.88 & 16.32 & -0.23 & 0.02 & -0.6 \\
1000 & 5.59 & 22.65 & 6.96 & 7.17 & 6.7 \\
1500 & 8.43 & 33.58 & 19.21 & 19.36 & 18.9 \\
2000 & 11.27 & 44.85 & 31.61 & 31.70 & 31.3 \\
2500 & 14.11 & 56.37 & 44.09 & 44.10 & 43.7 \\
3000 & 16.95 & 68.09 & 56.60 & 56.54 & 56.1 \\
3500 & 19.79 & 79.98 & 69.13 & 69.00 & 68.5 \\
4000 & 22.63 & 92.01 & 81.67 & 81.47 & 81.0 \\
\hline
\end{tabular}

${ }^{a}$ Experimental Gibbs energy changes for the $\mathrm{H}+\mathrm{CO} \leftrightarrow \mathrm{HCO}$ reaction retrieved from Chase (1998). ${ }^{(6)}$

b Theoretical Gibbs energy changes calculated from the ROCCSD(T)/CBS//UCCSD/cc-pVDZ combined treatment by Vichietti et al. (2019). ${ }^{(7)}$

Table S5. Entropies (in cal $\left.\mathrm{mol}^{-1} \mathrm{~K}^{-1}\right)$ relative to reactants $(\mathrm{H}+\mathrm{CO}$ ) for the stationary point structures related to the $\mathrm{H}+\mathrm{CO} \leftrightarrow \mathrm{HCO}$ reaction, as calculated at the CCSD(T)/CBS//CCSD/augcc-pVTZ level and different temperatures (in K), $T$.

\begin{tabular}{rcccc}
\hline \multicolumn{1}{c}{$T$} & PrC & TSRP & HCO & $\Delta S_{\exp }{ }^{\mathrm{a}}$ \\
\hline 50 & -5.43 & -12.79 & -14.01 & $\ldots$ \\
100 & -5.62 & -15.49 & -16.76 & -16.69 \\
200 & -5.66 & -17.77 & -19.50 & -19.44 \\
300 & -5.67 & -18.84 & -21.05 & -20.99 \\
500 & -5.68 & -20.01 & -22.73 & -22.67 \\
700 & -5.68 & -20.71 & -23.58 & -23.50 \\
1000 & -5.68 & -21.43 & -24.24 & -24.13 \\
1500 & -5.68 & -22.24 & -24.70 & -24.57 \\
2000 & -5.68 & -22.81 & -24.89 & -24.76 \\
2500 & -5.68 & -23.26 & -24.99 & -24.85 \\
3000 & -5.68 & -23.62 & -25.04 & -24.90 \\
3500 & -5.68 & -23.92 & -25.08 & -24.93 \\
4000 & -5.68 & -24.19 & -25.10 & -24.94 \\
\hline
\end{tabular}

${ }^{a}$ Experimental entropy changes for the $\mathrm{H}+\mathrm{CO} \leftrightarrow \mathrm{HCO}$ reaction retrieved from Chase (1998). ${ }^{(6)}$ 
Table S6. Forward (in $\mathrm{cm}^{3}$ particle $\mathrm{s}^{-1} \mathrm{~s}^{-1}$ ), $k_{\mathrm{f}}$, and reverse (in $\mathrm{s}^{-1}$ ), $k_{\mathrm{r}}$, ICVT/SCT rate constants encountered for the $\mathrm{H}+\mathrm{CO} \leftrightarrow \mathrm{HCO}$ reactions, as calculated at the $\mathrm{CCSD}(\mathrm{T}) / \mathrm{CBS} / / \mathrm{CCSD} / \mathrm{aug}$-ccpVTZ level and different temperatures (in K), $T$.

\begin{tabular}{ccc}
\hline$T$ & $k_{\mathrm{f}}$ & $k_{\mathrm{r}}$ \\
\hline 298 & $3.48 \times 10^{-13}$ & $1.28 \times 10^{0}$ \\
310 & $4.26 \times 10^{-13}$ & $4.18 \times 10^{0}$ \\
320 & $5.00 \times 10^{-13}$ & $1.05 \times 10^{1}$ \\
330 & $5.82 \times 10^{-13}$ & $2.50 \times 10^{1}$ \\
340 & $6.73 \times 10^{-13}$ & $5.68 \times 10^{1}$ \\
350 & $7.73 \times 10^{-13}$ & $1.23 \times 10^{2}$ \\
360 & $8.82 \times 10^{-13}$ & $2.57 \times 10^{2}$ \\
373 & $1.04 \times 10^{-12}$ & $6.30 \times 10^{2}$ \\
\hline
\end{tabular}

Table S7. Traditional and modified Arrhenius' parameters $\left[A, \beta\right.$, and $E_{\mathrm{ARR}}$ (in $\mathrm{kcal} \mathrm{mol}^{-1}$ )] for the $\mathrm{H}$ $+\mathrm{CO} \leftrightarrow \mathrm{HCO}$ reactions, which were fitted from 298 to $373 \mathrm{~K}$ from ICVT/SCT rate constants.

\begin{tabular}{cccccr}
\hline Reaction & Arrhenius & $A^{\mathrm{a}}$ & $\beta$ & $E_{\mathrm{ARR}}$ & $\mathrm{MAD}^{\mathrm{b}}$ \\
\hline $\mathrm{H}+\mathrm{CO} \rightarrow \mathrm{HCO}$ & Traditional & $7.936 \times 10^{-11}$ & 0.0000 & 3.22 & 0.7 \\
& Modified & $9.584 \times 10^{-12}$ & 1.9121 & 1.96 & $<0.1$ \\
& & & & & \\
$\mathrm{HCO} \rightarrow \mathrm{H}+\mathrm{CO}$ & Traditional & $3.091 \times 10^{13}$ & 0.0000 & 18.25 & 1.0 \\
& Modified & $1.571 \times 10^{12}$ & 2.6950 & 16.47 & $<0.1$ \\
\hline
\end{tabular}

a The $A$ values for $\mathrm{H}+\mathrm{CO} \rightarrow \mathrm{HCO}$ and $\mathrm{HCO} \rightarrow \mathrm{H}+\mathrm{CO}$ reactions are given, respectively, in units of $\mathrm{cm}^{3}$ particle ${ }^{-1} \mathrm{~s}^{-1}$ and $\mathrm{s}^{-1}$.

${ }^{b}$ MAD means maximum absolute deviations (in \%) found between rate constants obtained from Equation (6) in the main text and those presented in Table S6.

\section{REFERENCES}

(1) Song, L.; van der Avoird, A.; Groenenboom, G. C. Three-dimensional ab initio potential energy surface for H-CO $\left(\tilde{X}^{2} A^{\prime}\right)$. J. Phys. Chem. A 2013, 117, 7571-7579.

(2) Peters, P. S.; Duflot, D.; Wiesenfeld, L.; Toubin, C. J. The H $+\mathrm{CO} \leftrightarrow \mathrm{HCO}$ reaction studied by ab initio benchmark calculations. J. Chem. Phys. 2013, 139, 164310(1)-164310(15).

(3) Lide, D. R. CRC handbook of chemistry and physics, 84th ed.; CRC Press LLC: Boca Raton, 2004.

(4) Hirota, E. Third-order anharmonic potential constants and equilibrium structures of the formyl and hydroperoxyl radicals. J. Mol. Struc. 1986, 146, 237-252.

(5) Bishop, D. M.; Cheung, L. M. Vibrational contributions to molecular dipole polarizabilities. $J$. Phys. Chem. Ref. Data 1982, 11, 119-133.

(6) Chase Jr, M. W. NIST-JANAF thermochemical tables, 4th ed.; Chase Jr, M. W., Ed.; Gaithersburg, MD, 1998.

(7) Vichietti, R. M.; Spada, R. F. K.; da Silva, A. B. F.; Machado, F. B. C.; Haiduke, R. L. A. A proposal for the mechanism of the $\mathrm{CH}+\mathrm{CO}_{2}$ reaction. ACS Omega 2019, 4, 17843-17849. 